
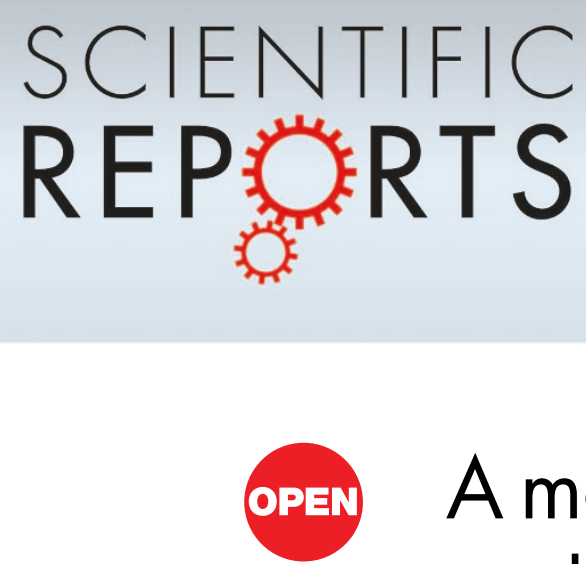

\title{
A mathematical model of the London riots and their policing
}

SUBJECT AREAS: APPLIED MATHEMATICS COMPUTATIONAL SCIENCE APPLIED PHYSICS PSYCHOLOGY AND BEHAVIOUR

Received

11 October 2012

Accepted

4 February 2013

Published

21 February 2013

Correspondence and requests for materials should be addressed to T.D. (toby.davies.09@ ucl.ac.uk)

\author{
Toby P. Davies ${ }^{1,2}$, Hannah M. Fry ${ }^{1,3}$, Alan G. Wilson ${ }^{3}$ \& Steven R. Bishop'
}

'Department of Mathematics, University College London, Gower Street, WC1E 6BT, ${ }^{2}$ Department of Security and Crime Science, University College London, Gower Street, WC1E 6BT, ${ }^{3}$ Centre for Advanced Spatial Analysis, University College London, Gower Street, WC1E 6BT.

In August 2011, several areas of London experienced episodes of large-scale disorder, comprising looting, rioting and violence. Much subsequent discourse has questioned the adequacy of the police response, in terms of the resources available and strategies used. In this article, we present a mathematical model of the spatial development of the disorder, which can be used to examine the effect of varying policing arrangements. The model is capable of simulating the general emergent patterns of the events and focusses on three fundamental aspects: the apparently-contagious nature of participation; the distances travelled to riot locations; and the deterrent effect of policing. We demonstrate that the spatial configuration of London places some areas at naturally higher risk than others, highlighting the importance of spatial considerations when planning for such events. We also investigate the consequences of varying police numbers and reaction time, which has the potential to guide policy in this area.

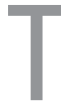
he need for public policy to be informed by an evidence-based approach has been recognised for some time; however, this is often problematic, particularly in the context of rare events. The difficulty is especially evident in situations where quantitative recommendations are required, such as estimating the appropriate contingency for a certain scenario, since traditional hypothesis testing is undermined by the paucity of data. In such cases, mathematical modelling has much to offer, allowing rigorous quantitative analysis of the system in question and the testing of varying scenarios. Recent advances in the modelling of large-scale social systems using techniques of complexity science mean this is now a viable approach, and indeed its potential has been demonstrated in such fields as epidemic modelling ${ }^{1,2}$, crowd control $^{3}$ and infrastructure resilience ${ }^{4}$. Here, we employ such an approach in the context of the 2011 London riots and the policy questions subsequently arising.

The London riots occurred between 6-10 August 2011, as the UK experienced its most widespread and sustained period of civil unrest for at least 20 years. Repeated episodes of looting, rioting, arson and inter-personal violence took place in several cities, including London, Manchester and Birmingham. The consequences of the events include numerous instances of injury, including five deaths, and extensive property damage, for which

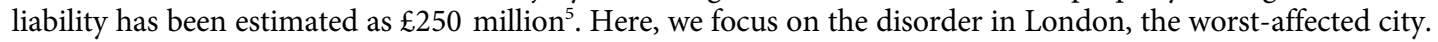

The London riots have been the subject of much research in the academic ${ }^{6}$, governmental ${ }^{7}$ and journalistic ${ }^{8}$ communities. The majority of this research, however, as with much of that considering previous episodes ${ }^{9}$, has focussed on the psycho-social motives of individual rioters, ascribing willingness to participate to various social factors, including unemployment, poor police relations and endemic criminality. Our work is distinct from this; rather than consider how and why the riots began, we take their initiation as our starting point and instead consider their spatio-temporal development. This approach is informed by the policy question which motivates our work: how, once such an incident is in progress, the police might best respond in order to suppress disorder as quickly as possible.

Several questions relating to the response of the authorities were raised following the disorder. Although order was restored after five days, it has been variously claimed that the police were inadequately prepared and slow to react to developments, and that disorder might have been suppressed sooner; indeed, official inquiries have acknowledged such shortcomings ${ }^{5,10,11}$. Alongside a need to anticipate better the disorder itself, these inquiries have emphasised the need to establish a level of policing resource, and mode of response, commensurate with an outbreak of this magnitude. Mathematical modelling can contribute to this by allowing quantitative examination of the effect of varying police responses, and the investigation of a range of scenarios.

A crucial factor in determining police response to a riot, and indeed for policing in general ${ }^{12}$, is an understanding of the spatio-temporal distribution of events. The London riots are notable for the fact that, despite being apparently catalysed by a specific incident - the fatal shooting by a police officer of a suspect in Tottenham, North 
London, and a subsequent peaceful protest - disorder escalated in a dramatic and unanticipated way, spreading widely across the city. Understanding why and how this spreading occurred, and why some areas were afflicted more than others, is therefore fundamental to the planning of responses.

The use of mathematical modelling to explore the mechanisms behind the spatial heterogeneity seen in the disorder can go some way towards explaining the events. In this respect, our work follows the 'generative' approach previously used in the modelling of, for example, residential segregation ${ }^{13}$, state formation ${ }^{14}$ and collective action in social networks ${ }^{15}$, in that our aim is simply to establish whether our hypothesised mechanisms are capable of giving rise to realistic patterns. Although our work is motivated by empirical data, we make no attempt to replicate the London disorder, but rather to imitate the general 'stylised facts' observed.

A significant body of theory exists concerning criminal involvement and target choice, which, in tandem with the empirical results presented in the following section, informs the model. Although such theories are premised on the assumption that offenders act rationally during a riot, and so cannot necessarily be invoked a priori, previous research suggests that, in general, that assumption is defensible ${ }^{16-18}$. Indeed, research specific to the London disorder suggests that criminological theory, such as social disorganisation theory ${ }^{19}$ and rational choice theory ${ }^{20}$, is applicable in that case ${ }^{21}$, and that its implications are therefore a well-justified basis for a model.

The concept of the rational offender implies that their decisions are influenced by the relative merits of actions - for instance, the potential rewards available at different sites - and that they seek to minimise costs, choosing nearby targets and seeking to avoid capture, as appears to be the case during rioting ${ }^{22}$. From a modelling perspective, this implies that these factors should be included in any formulation of the utility associated with a particular location. Closely related to this, crime pattern theory ${ }^{23}$ seeks to explain the location of crime in terms of the 'awareness spaces' of potential offenders, i.e. the locations of opportunities and offenders' knowledge of them. For a riot, this would again suggest that participants are more likely to target nearby locations, as has been demonstrated across a variety of crime type ${ }^{24}$, and that these locations are likely to be those which are commonly perceived to offer large potential rewards. These ideas inform our choice of spatial system in the model.

Considering instead the initial decision to offend, theory can inform both the mechanism by which potential participants are influenced, and the differing effect this influence might have. Theories of environmental criminology state that environmental precipitators (in this case, knowledge of ongoing rioting) can serve to prompt, pressure, permit or provoke offending ${ }^{25}$; the 'safety in numbers' effect in riots, in terms of the risk of arrest ${ }^{26,27}$, is a particularly clear example of how this might modify the cost/benefit structure $^{28}$. In the case of London, there is a widely-held perception ${ }^{6}$, that awareness of disorder provided a self-reinforcing stimulus to rioter involvement, facilitated in many cases by social media. These ideas are clearly fundamental to the evolution and spreading of disorder, and suggest a contagion-like mechanism for this.

Such cues may not, however, act uniformly, and whether they lead to offending may be dependent on local circumstances. The notion that environment affects the propensity of residents to engage in crime is well-developed in criminology: social disorganisation theory ${ }^{19}$ suggests that criminality is more likely to take hold in areas with weak social fabric, due to a lack of informal social control ${ }^{29}$. Deprivation relates closely to these ideas: more deprived communities lack the resources and structure to regulate themselves in this way. For the purpose of model-building, this implies a need to incorporate geo-demographic factors in the proposed mechanisms.

Previous attempts to model riots have employed both continuous and agent-based approaches. The former have generally been attempts to adapt models of crowd dynamics to the case of rioting whilst doing little to accommodate realistic human behaviour ${ }^{30,31}$. Agent-based models, following the civil violence model of Epstein $^{26}$, have had some success in using game-theoretic concepts to inform agent behaviour ${ }^{32,33}$. These have generally, though, given little attention to geographical concerns, either treating movement as random or else in a fairly naive sense. More recent approaches, however, have remedied this somewhat by incorporating real spatial data via GIS ${ }^{34}$ and including sophisticated spatial decision-making ${ }^{35}$. Our work is, as far as we are aware, the first to incorporate such behaviour outside an agent-based framework, and is also differentiated by its focus on the case of London, both in its incorporation of data and consideration of particular policy concerns.

After describing the general trends observed in the data, we describe a model which incorporates several phases of riot development: a contagious process of involvement, a target choice stage, and an interaction between participants and police. We then demonstrate, via numerical simulation, that the model is capable of reproducing the general trends identified. With a realistic simulation established, this framework is used to explore policy issues, such as the effect of varying police response and the pre-disposition of certain areas to riot activity.

\section{Results}

Characteristics of disorder. In seeking insight into the behaviour of individuals during an episode of rioting, we consider both existing theoretical research into such incidents ${ }^{36}$ (and criminal activity in general) and specific observations from the London disorder. The latter takes the form of analysis of data provided by the Metropolitan Police, which contains the details of all individuals arrested in relation to the riots and matches the home addresses and offence locations of suspects. Since it is typically argued that individuals act rationally during a riot (i.e. that their decisions are based on some cost/benefit analysis) ${ }^{16-18}$ these observations can be used to inform a model of the actions of rioters.

A fundamental observation is the predominant targeting of retail sites, reflecting the acquisitive nature of much offending. Crimes against commercial premises, including both acquisitive crime and criminal damage, accounted for $51 \%$ of all offences in the UK as a whole $^{37}$, and offences clustered in areas such as Clapham Junction, Croydon, Ealing and Brixton. This can be immediately reconciled with crime pattern theory ${ }^{23}$; the richness of opportunity at retail premises is likely to be common knowledge amongst riot participants, and they therefore act as crime attractors. In line with this, for our model we adopt a system of retail centres as the sites of disorder.

We also consider the origins of offenders, i.e. the locations of their residences, and, therefore, the distances they travelled to the sites where they offended. As seen in Figure 1a, the flows of offenders follow a clear distance-decay relationship. Although statistical tests ${ }^{38}$ find that the distribution does not correspond to most common forms, the best fit is provided by an exponential distribution with parameter 0.274. An offender's perception of distance does not necessarily aggregate to an exponential distance decay, since other factors, some of which are temporally-varying, are likely to contribute, and we nevertheless incorporate an exponential distance decay within in our model. Distributions such as these are reminiscent of those seen in the analysis of flows in retail systems ${ }^{39,40}$, and so, noting also the central role of commercial centres, we model the behaviour of rioters partly by analogy with this.

Analysing the riot locations further, we explore the relationship between deprivation and offending. Figure $1 \mathrm{~b}$ shows that a disproportionately high number of offences occurred in more deprived areas (approximately 50\% within the 20\% most deprived), using the UK's Index of Multiple Deprivation (IMD) to rank census units. Looking instead at suspects' residences, Figure 1c shows the average 


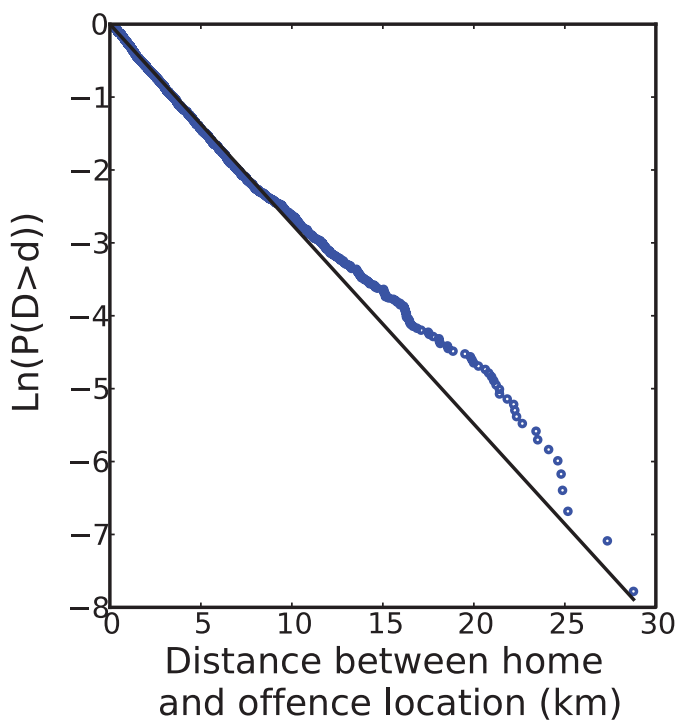

(a)

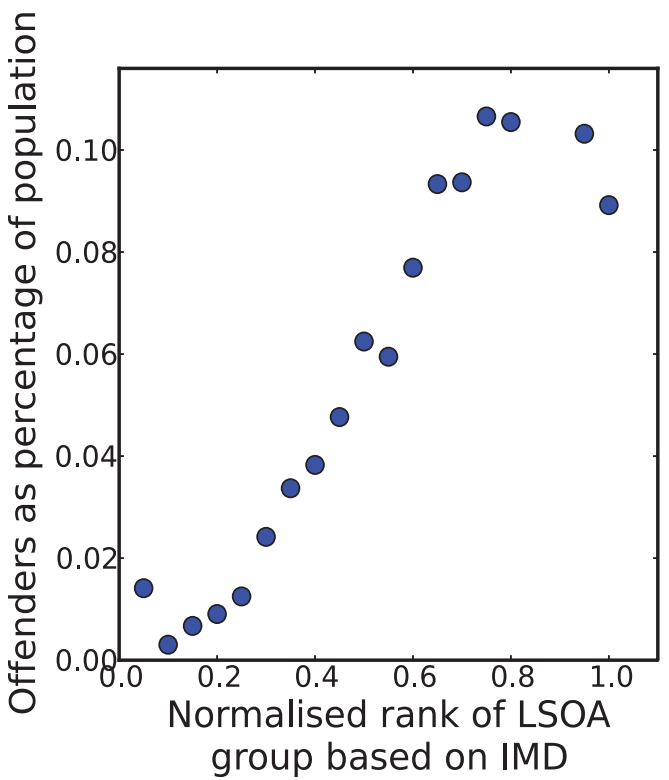

(c)

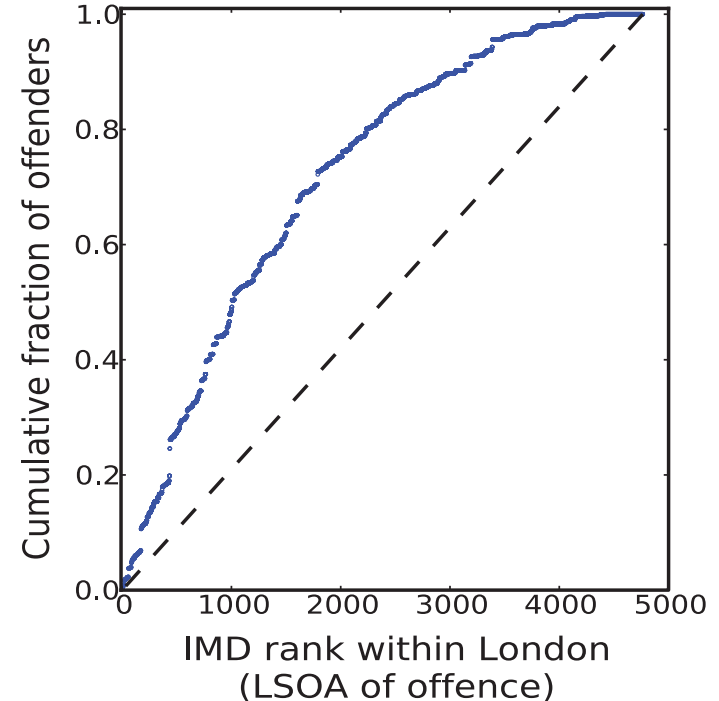

(b)

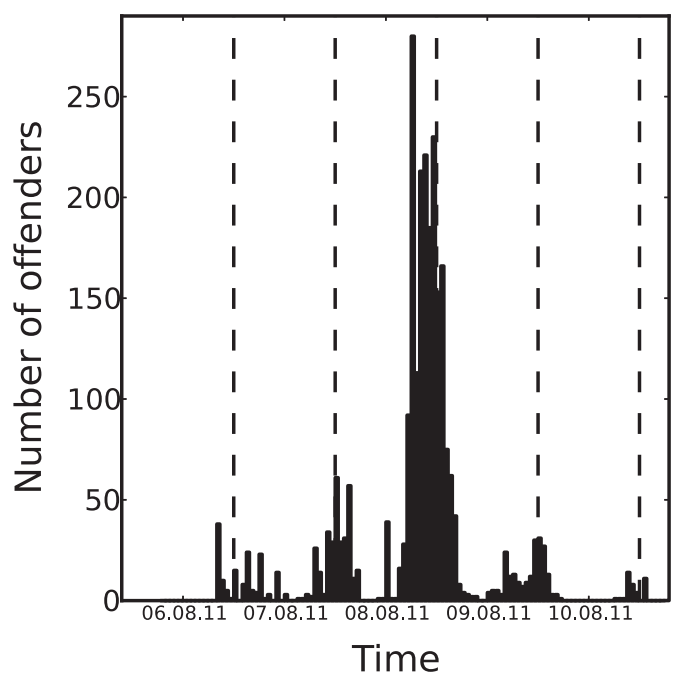

(d)

Figure 1 Observations from arrest data. (a) Log-linear plot of the complementary cumulative distribution function of $D$, the distance between residential and offence locations. The straight line shows a hypothetical exponential distribution with parameter 0.274 ( \pm 0.01 for a $95 \%$ confidence interval), for which the Kolmogorov-Smirnov distance statistic is 0.0246 (which compares with 0.332 for the equivalent fitted power-law). (b) Lorenz curve for the distribution of riot locations amongst Lower Super Output Areas (LSOAs; UK census units with average population approximately 1,500) ranked according to deprivation (where 1 is most deprived). The dashed line represents perfect equality. (c) Relationship between area-level deprivation and the proportion of residents involved in disorder, where the horizontal axis represents a score derived from IMD so that all values lie in $[0,1]$ and so that London's most deprived area is given a value of 1 . d) Temporal distribution of recorded crime.

proportion of riot suspects for groups of LSOAs ordered by deprivation, where in this case, anticipating its incorporation as a variable in the model, we use a deprivation score based on IMD ranking. A relationship between offending and deprivation has also been found elsewhere ${ }^{37}$, and youth unemployment and child poverty have also been identified ${ }^{41}$. That the most deprived areas acted disproportionately as both origins and destinations will clearly influence the distance distribution, and vice versa, but work elsewhere shows that both effects persist when controlling for the other ${ }^{21}$.

With this notion in mind, we incorporate the deprivation score discussed above as a feature within our model, allowing for a higher probability of offending in deprived areas.

We also note distinctive temporal patterns in the riot data, as seen in Figure 1d. From the small initial disturbance, incidents escalated in volume and intensity on each successive day, with police response growing in line with this, from 3,480 on Saturday evening to 16,000 by Tuesday ${ }^{5}$. This may also be seen at the scale of individual days, where the majority of criminality took place at night and built to a peak in the early hours. Whilst various explanations for this have been put forward, a particularly compelling one suggests that awareness of disorder provided a self-reinforcing stimulus to rioter involvement ${ }^{6}$, and a contagion-based model is therefore appealing.

Model. We develop a mathematical model with the aim of exploring the spatial and temporal patterns of the events in London. Recognising that non-linearities inherent in the system imply a significant dependence on initial conditions (which are unknown), and that numerous factors not considered here are likely to play a 
material role, we do not seek to replicate exactly the London events. Rather, we aim to produce a 'generative' type model which can give rise to realistic patterns and macro-level behaviour that insight might plausibly be gained through analysis of the underlying dynamics.

Our model draws on elements of several existing ones; our contribution is in their combination and adaptation to produce an integrated spatial model of disorder, and in the analysis of varying police strategies. The model can be divided into three components: an epidemiological model for riot participation, a spatial interaction model (SIM) ${ }^{42}$ for the spatial allocation of rioters and police, and a model for interaction between rioters and police previously applied in the context of civil violence ${ }^{26}$.

General concepts. The model is defined across a discrete system of two entities: residential areas and retail centres. These are indexed by $i$ and $j$ respectively and embedded in space, and we use LSOAs and defined 'retail cores' when considering London. Participating individuals are notionally tracked through the system via a logical sequence which involves a decision to participate taking place at their home, a choice of site at which to offend, and possible removal due to arrest by police officers. These officers are active at all times but may move and be located according to different principles.

To model rioters' decisions, some concept of the attractiveness of a riot site is required. This is formulated using a 'cost/benefit' structure, as is normal for SIMs, where benefit represents the potential reward at a site and cost embodies both travel cost and the deterrent effect of police.

We assume that the benefit for site $j$ is given by the logarithm of $Z_{j}$, a non-dimensional measure of its relative value (e.g. the ratio of $j$ 's floorspace to the mean across the system), as is a standard assumption in retail models of this type ${ }^{43,44}$, reflecting diminishing returns to scale. For $b_{i j}$, the benefit of site $j$ as perceived by an individual in $i$, we therefore have

$$
b_{i j} \sim \log Z_{j}, \quad \forall i .
$$

Turning to deterrence, we suggest that the primary gauge by which an individual assesses whether the situation at a site is conducive to riot is the probability of arrest, determined by the relative numbers of rioters and police: low perceived chance of capture encourages participation. Several such expressions for probability of arrest have been proposed; in this case we take an adapted version of the formulation of Epstein ${ }^{26}$ as our starting point:

$$
\mathrm{P}(\text { arrest in } j \text { in one time unit })=1-\exp \left(-\left\lfloor\frac{Q_{j}}{a D_{j}}\right\rfloor\right),
$$

where $Q_{j}$ is the number of police officers in $j, D_{j}$ the number of rioters in $j$, and $a$ the number of police officers required, on average, to 'contain' one rioter. The use of the floor function $\left\lfloor Q_{j} / a D_{j}\right\rfloor$ has empirical motivation; the Metropolitan Police review of the London disorder ${ }^{11}$ explicitly states that "decisions were made not to arrest due to the prioritisation of competing demands...specifically, the need to protect emergency services, prevent the spread of further disorder and hold ground until the arrival of more police resources". Accordingly, when the police are 'outnumbered' at a site (i.e. $Q_{j}<a D_{j}$ ), the situation is considered to be out of control and the police are unable to make any arrests without the addition of 'backup' (and thus the probability is 0 ). On the basis that increased probability corresponds to increased deterrence, we therefore express deterrence thus:

$$
\text { deterrence } \left.\sim \frac{Q_{j}}{a D_{j}}\right\rfloor .
$$

We also incorporate a linear function of the distance between residential areas and riot sites, as is typical for analogous retail systems.
Taking this as proportional to $d_{i j}$, the distance between the centroids of $i$ and $j$, we can then combine with (2) and (3) to obtain the full expression for benefit - cost:

$$
w_{1} \log Z_{j}-w_{2} d_{i j}-w_{3}\left\lfloor\frac{Q_{j}}{a D_{j}}\right\rfloor,
$$

where the $w_{n}$ are constants. The associated attractiveness term $W_{i j}$ which appears in the terms of the spatial interaction model can, as described elsewhere ${ }^{44}$, then be written as follows:

$$
W_{i j}=Z_{j}^{\alpha_{r}} \exp \left(-\beta_{r} d_{i j}\right) \exp \left(-\left\lfloor\frac{\gamma_{r} Q_{j}}{D_{j}}\right\rfloor\right)
$$

where $\alpha_{r}, \beta_{r}$ and $\gamma_{r}$ (which itself absorbs $a$ ) are parameters to be obtained in calibration with real-world data (the subscript $r$ denoting reference to riot participants). It is through the form of (5) that an exponential distance decay, discussed in the previous section, features in the model.

Riot participation. Motivated by the hypothesis, consistent with the temporal progression of the riots, that exposure to nearby disorder had the effect of inciting participation, we propose a SusceptibleInfected-Removed (SIR) model $^{45}$; that is, a mechanism akin to infection by which individuals transfer to an active rioting state according to their level of exposure. Recalling the correlation between propensity to riot and deprivation, we also incorporate this, and the function we propose is therefore:

$$
\mathrm{P}(\text { individual in } i \text { chooses to offend })=\rho_{i}^{\mu} \frac{\sum_{j} W_{i j}}{1+\sum_{j} W_{i j}},
$$

where $\rho_{i}$ is a measure of the deprivation in $i$ (which we take to be based upon the IMD) and $\mu$ an exponent to be calibrated. A logistic function is used here to represent the existence of a threshold at which rioting becomes appealing; any transition is likely to be localised rather than gradual. Intuitively, this probability will be small when the overall attractiveness of potential riot areas is low, whereas, when the 'ambient' level of rioting is high, the probability of offending tends towards $\rho_{i}^{\mu}$. From another perspective, where two areas were equally exposed to disorder, greater participation would arise in the more deprived of the two.

Translating this to the macro-level for a residential area $i$, we therefore find an expression for $N_{i}(t)$, the rate at which individuals choose to participate at time $t$. Under the assumption that decisions are independent between individuals, this is given by the product of population size and decision probability,

$$
N_{i}(t)=\eta I_{i}(t) \rho_{i}^{\mu} \frac{\sum_{j} W_{i j}(t)}{1+\sum_{j} W_{i j}(t)},
$$

where $\eta$ is an infection rate and $I_{i}(t)$ the number of inactive individuals resident in area $i$. We can now formulate expressions for $I_{i}(t)$ and $R_{i}(t)$, the number of rioters whose residence is in a given zone $i$, as well as their change in a time period $[t, t+\delta t)$. These, along with their initial conditions $\left(I_{i}(0)\right.$ is the residential population of $i$ and $R_{i}(0)$ a seed of participants, to be chosen) determine the numbers of individuals of each type, in each residential area, at all times. The choice to structure the model in this way is motivated by our focus on the residential origins of rioters, since it enables us to understand the composition of rioting groups in these terms. At this stage we also include an extra term $C_{i}(t)$, to be fully defined later, for the rate at which participants from $i$ are arrested at time $t$ :

$$
R_{i}(t+\delta t)=R_{i}(t)+\delta t\left(N_{i}(t)-C_{i}(t)\right)
$$




$$
I_{i}(t+\delta t)=I_{i}(t)-\delta t N_{i}(t)
$$

Spatial assignment. We assign active rioters to sites of disorder using an entropy-maximising SIM; the purpose of these models is to estimate the most probable flows in a spatial system such as ours, given certain constraints ${ }^{42}$.

Rather than incorporating the attractiveness function, $W_{i j}$, directly into the spatial interaction equations, we use its moving average over a number of previous time steps, for several reasons: to account for factors such as travel time on the part of rioters, to represent 'lag' in the spread of information through the system, and to dampen the effect of sudden fluctuations in attractiveness. The values used to determine the assignments at a given time, referred to as effective attractiveness and denoted $W_{i j}^{e}$, are therefore the average values of $W_{i j}$ over the $L_{r}$ most recent time steps in our discretised temporal scheme (which has intervals $\delta t$; when $t<\left(L_{r}-1\right) \delta t$, we 'pad' with the $t=0$ value):

$$
W_{i j}^{e}(t)=\frac{1}{L_{r}} \sum_{l=0}^{L_{r}-1} W_{i j}(t-l \delta t) .
$$

Following the standard entropy maximising derivation of a $\mathrm{SIM}^{44}$, it can be shown that $S_{i j}$, an estimate of the number of rioters from $i$ who are participating in disorder in $j$ at time $t$ is given by:

$$
S_{i j}(t)=\frac{R_{i}(t) W_{i j}^{e}(t)}{\sum_{k} W_{i k}^{e}(t)} .
$$

An identical expression for $S_{i j}$ may be formulated using an alternative derivation: by considering (4) as a utility term in a conditional logit mode ${ }^{46}$. In either case, summing over residential areas $i$ yields the total number of rioters $D_{j}$ in $j$ :

$$
D_{j}(t)=\sum_{i} \frac{R_{i}(t) W_{i j}^{e}(t)}{\sum_{k} W_{i k}^{e}(t)} .
$$

It should be noted here that each time unit is therefore implicitly defined as the mean time taken for each participant to travel from a home location to a chosen riot site.

The assignment of police resources to areas of disorder is also realised via a SIM, as for riot participants; there are, however, noteworthy differences. First, police units have no 'home' location and are active and situated at potential sites of disorder at all times. The response lag $L_{p}$ is also different to that for rioters (and intended to be higher); reflecting the delay in learning of the plans and movements of rioters, and conferring upon the rioters a degree of 'firstmover advantage'.

The main difference for police, however, is in the attractiveness function, analogous here to the requirement for officers at a given site. Following a similar argument to that of the rioters seen in (4), we assume the benefit - cost of police follows:

$$
\bar{w}_{1} \log Z_{j}+\bar{w}_{2} D_{j}
$$

This expression (13) includes no spatial decay term, reflecting the fact that the police do not prioritise incidents on the basis of proximity ${ }^{10}$ and can travel to incidents rapidly. In addition, the second term is a function of rioter numbers only: given that their aim is to eliminate all disorder, the number of police already at a site is likely to be immaterial to the police. As in (5) and described elsewhere ${ }^{44}$, the attractiveness function $V_{j}$ representing police requirement, is therefore:

$$
V_{j}=Z_{j}^{\alpha_{p}} \exp \left(\gamma_{p} D_{j}\right)
$$

where $\alpha_{p}$ and $\gamma_{p}$ are, as before, parameters to be calibrated which encode the relative importance of the two factors. Following the identical process seen with (5) above, we may first calculate effective requirement to take into account time lags in the system,

$$
V_{j}^{e}(t)=\frac{1}{L_{p}} \sum_{l=0}^{L_{p}-1} V_{j}(t-l \delta t) .
$$

and, in conjunction with a SIM, as in (11) and (12), can derive an expression for the total number of police officers in location $j$ at time $t$ :

$$
Q_{j}(t)=P \frac{V_{j}^{e}(t)}{\sum_{k} V_{k}^{e}(t)},
$$

where $P$ is the total number of police officers in the system.

Interaction between police and rioters. To model the interaction of police and rioters, we return to the mechanism of arrest and its associated probability described previously. This gives the probability of capture for an individual rioter, and multiplying by the number of participants present therefore gives the expected number arrested. Since, for reasons explained previously, we classify participants by residential location, this is done separately for each area to give $C_{i}(t)$, the rate at which individuals who originated in $i$ are arrested at time $t$ :

$$
C_{i}(t)=\tau \sum_{j} S_{i j}(t)\left(1-\exp \left(-\left\lfloor\frac{Q_{j}}{D_{j}}\right\rfloor\right)\right) .
$$

where $\tau$ is an arrest rate parameter.

Demonstration case. As a step towards verification of the model, and to establish a 'base case' for further investigation, a series of numerical simulations were run, representing the escalation of events during a typical evening. Individual simulations ran for 10 time units (where one unit is the time taken for a rioter to travel to their destination) and involved sequential iteration through the model equations in the order (12), (16), (8). The system was seeded with 100 riot participants, assigned to residential areas in proportion to population and allocated to sites of disorder according to the static component of attractiveness (i.e. $Z_{j}^{\alpha_{r}}$ ). Similarly, 5,000 police officers (the approximate number deployed on each of the first 3 days in London) were initially placed at retail sites according to $Z_{j}^{\alpha_{p}}$. Given the high dimensionality of the model, many parameter sets were found to yield feasible results. To focus our discussion an example parameter set was chosen (Table 1) which gives rise to outcomes broadly in agreement with the features observed in the data, both in terms of borough level participants (Figure 2) and distance decay (Figure 3).

Since the riots occurred over 5 days, with incidents initialised in various locations across that period, these aggregate results offer little validation other than to confirm that the model is capable of replicating the general characteristics of the data. By instead initialising small incidents at just two locations, rather than simultaneously

Table 1 | Parameters used in base case simulation

\begin{tabular}{lccccccccccc} 
Parameter & $\alpha_{r}$ & $\beta$ & $\gamma_{r}$ & $\alpha_{p}$ & $\gamma_{p}$ & $\eta$ & $k$ & $\tau$ & $L_{r}$ & $L_{p}$ & $\delta t$ \\
\hline Value & 0.6 & 0.5 & 0.11 & 0.65 & 0.012 & 0.006 & 6 & 0.75 & 30 & 60 & 0.0143 \\
\hline
\end{tabular}




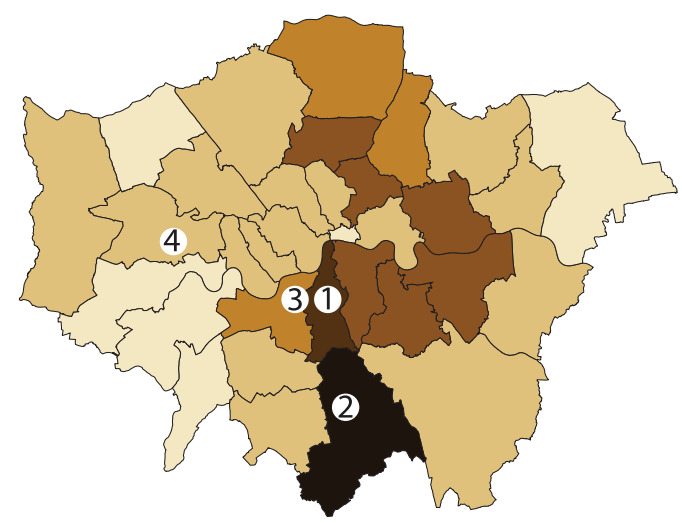

(a)

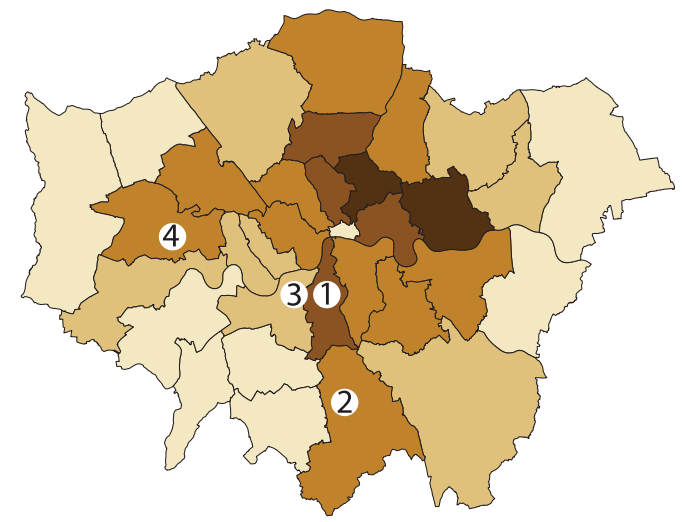

(b)
Percentage of rioters

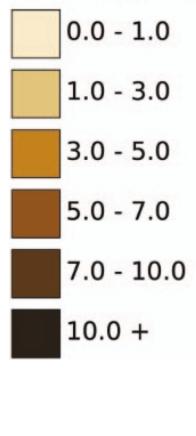

(c)

Figure $2 \mid$ Borough level choropleth of rioter residential locations from (a) data and (b) simulated results. Although the extreme dependence on initial conditions precludes our model from generating an exact replica of the observed incidents, the results show good qualitative agreement, with 26 of the 33 boroughs showing rioter percentages in the same or adjacent bands as the data. The remaining discrepancy may be accounted for by factors specific to the London disorder, such as communication between groups, other activity patterns occurring at the time, or social factors beyond the scope of this work. The labels 1,2,3,4 correspond to retail centres in Brixton, Croydon, Clapham Junction and Ealing respectively, which are considered individually in our later simulations.

across the city, we may explore the susceptibility of retail centres. Such initialisation is also reflective of the way in which real incidents are thought to arise: many of the outbreaks began as small local gatherings of unrest ${ }^{7,11}$.

Our analysis considers retail sites which were worst affected: Brixton, Croydon, Clapham Junction and Ealing. We ran four such simulations in each case, pairing the site of interest with each of its closest geographical neighbours. In all simulations (Figure 4) the centres which experienced widespread rioting in reality also saw substantial growth from the initial small disturbance in the model, while the vast majority of other retail locations saw incidents decay to zero. These results serve as further validation, but also, given the structure of the model, offer insight into why some sites were more susceptible than others, since the dynamics are based on a combination of factors: proximity to populous areas of high deprivation, and the balance of centre size and police presence. These are important results, as such an approach might be applied as an indicator of future susceptibility.

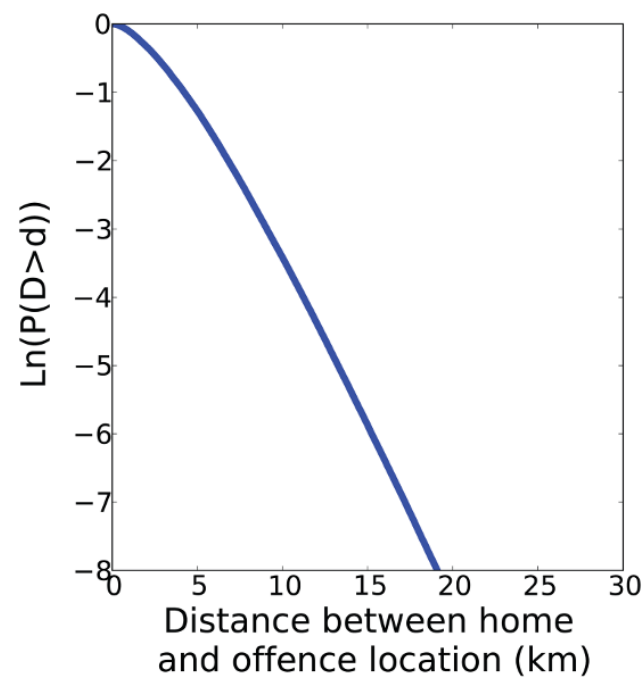

Figure 3 Log-linear plot of the complementary cumulative distribution function for $D$, the distances between residences and offence locations within the demonstration simulation.
Police resources and response. To gain quantitative insight into the level of police resource required to maintain control in a situation such as London's, we used the results of our demonstration case to analyse the effect of policing configuration on the development of disorder. To meaningfully compare realisations of the system, we define a quantity severity to summarise the cumulative disorder, given by the overall extent to which police are outnumbered by rioters:

$$
\text { severity }=\sum_{j} \sum_{t}^{T} \frac{D_{j}(t)}{Q_{j}(t)}
$$

Two parameters were varied independently in our simulations - total police $P$ and response lag $L_{p}$ - with parameters as in Table 1 otherwise, and results are shown in Figure 5. Police numbers correspond to those seen in London, and reflect what was seen in data: numbers above approximately 10,000 appear sufficient to suppress disorder. In the case of speed of response, the difference in severity as $L_{p}$ increases, relative to a base case of $L_{p}=0$, is plotted. After a noisy stage at small values, the severity appears to increase with lag. Although the increase is small as a proportion of absolute value, it should be borne in mind that these simulations are run with parameter values chosen such that a certain level of severity is assumed. Any changes, therefore, are variations around a level which has been implied a priori by other factors, such as police and rioter numbers. As expected, the trend observed reflects the importance of delivering police to scenes of disorder before control is lost. The same simulations were also run for other police configurations - specifically where police are assigned to locations initially, either uniformly or proportionally with $Z^{\alpha_{p}}$, and remain static throughout - but results differ only slightly from the dynamic case and are not shown.

\section{Discussion}

Motivated by the events in London in 2011, we have presented a model of civil disorder which is able to replicate the general features of that outbreak. Whilst simple, the model incorporates the fundamental features of such an incident, and might be applicable to others of a similar nature. We have used the model to explore how the level of resource available to be deployed by the police might affect the outcome of such an incident, which is currently an open question in the UK and has clear and timely implications for policy. The availability and use of police intelligence as a means of deploying 


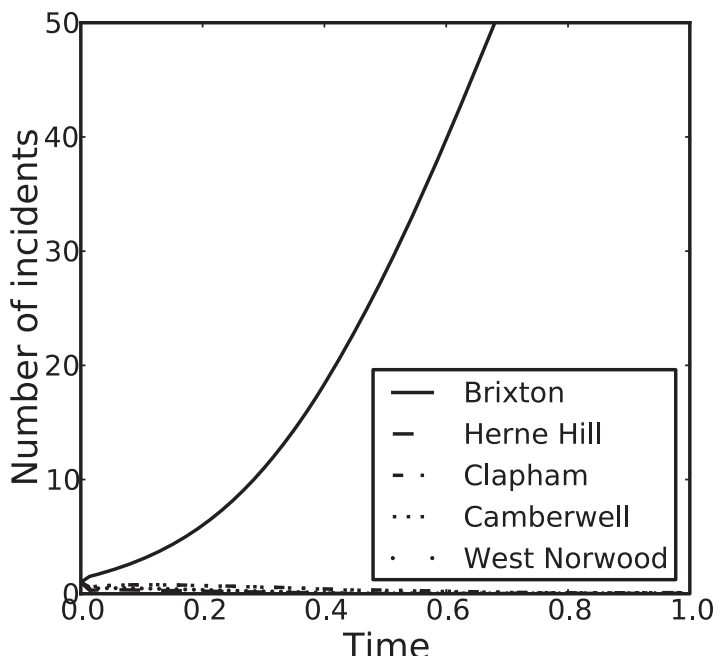

(a)

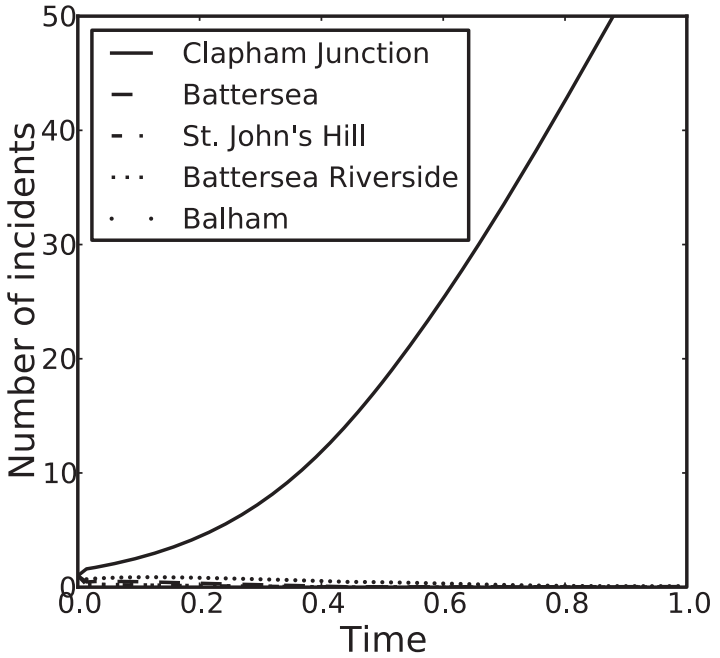

(c)

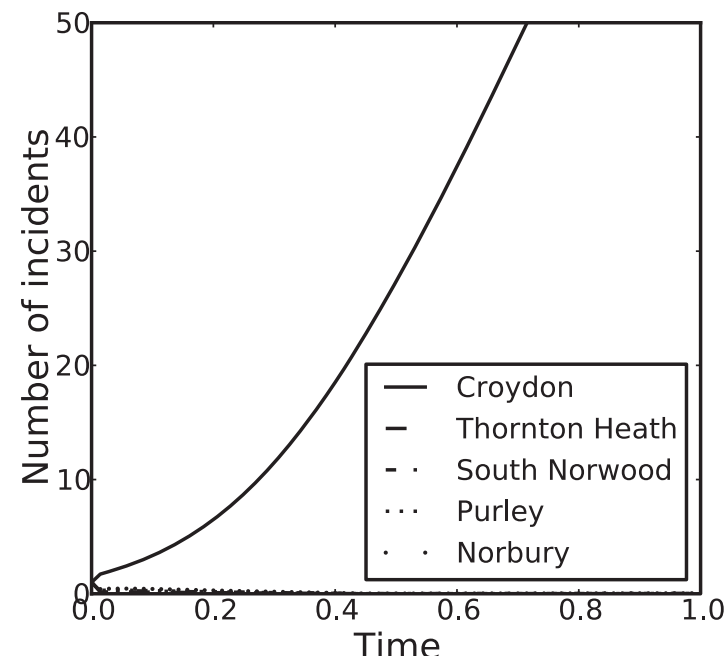

(b)

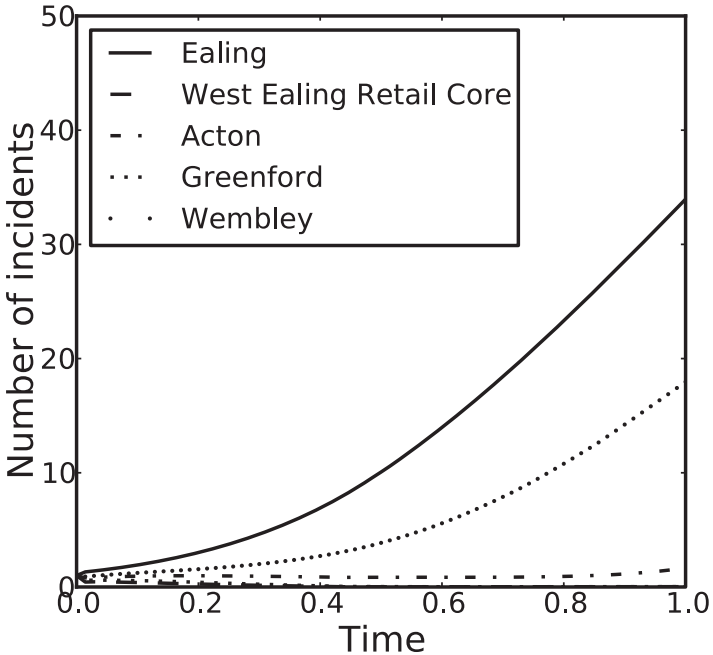

(d)

Figure $4 \mid$ The susceptibility of retail sites. For each of the four centres worst affected in the riots: (a) Brixton, (b) Croydon, (c) Clapham Junction and (d) Ealing, we ran four separate simulations, pairing the site of interest with each of its closest geographical neighbours in turn. An initial disturbance of one rioter was included at both sites and the model run to allow the incidents to evolve. Results shown for the sites of interest are the average of their four simulations, and in each case substantial growth is seen, particularly in comparison to the neighbouring centres.

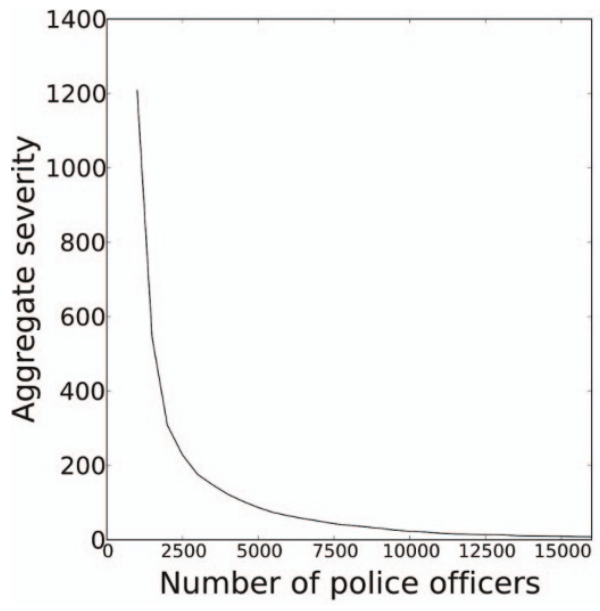

(a)

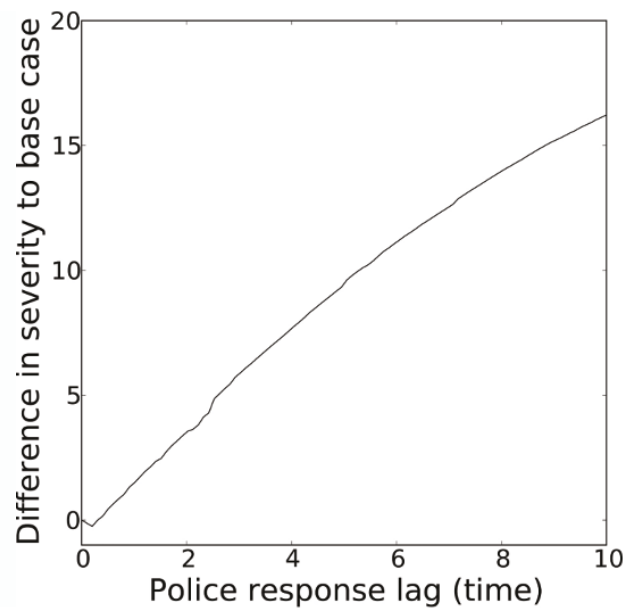

(b)

Figure $5 \mid$ The effect on severity of modifying (a) the number of police officers, and (b) their response lag. 
officers rapidly and efficiently - for which our concept of lag is a proxy - is also of particular interest. Both of these are found to have a material, and quantifiable, effect in our work. More sophisticated strategies on the part of both police and rioters could be incorporated in future iterations of the model, with the ultimate aim of making further quantitative recommendations.

\section{Methods}

Data. The behaviour of rioters is studied via the analysis of recorded crime data provided by the Metropolitan Police. This consists of all offences detected by the police in London in the period 6-11th August 2011, and which have been classified as being associated with the riots. Although the usual caveats concerning recorded crime data - regarding completeness and representativeness - do apply, these data are the best available for the London riots. Each record corresponds to a single incident, and specifies the area where the offence took place, the date and time at which the offence was estimated to have occurred, the residence of the offender and the age of the offender. It should be noted that no offender appears more than once in the data. This is preferable, since our focus is on involvement only, and the fact that a single instance of participation might comprise several crimes (perhaps determined subjectively) is liable to introduce bias to the data.

The dataset is comprised of 3,914 records; however, only 2,299 of these contain entries for both residential and offence location. Since both of these elements are fundamental to the model, only those records were analysed. In using this information to determine the distribution of 'journeys to crime' (i.e. the distance between home and offence), the Euclidean distance between the centroids of the basic census units within which the two points fall is used. Although it is recognised that this is not necessarily a true representation of the cost associated with travel, it is the only metric which can be applied consistently to our data; furthermore, common alternatives incorporating travel time may well not apply in such extraordinary circumstances.

The geographical data we use in our analysis of residential areas are Lower and Medium Super Output Areas, a hierarchical geographical structure defined by the UK government for census purposes. The census itself is also used to provide the residential populations of each of these areas, as used in the model. Government statistics for deprivation are also available at the level of Lower Super Output Areas, in the form of the Index of Multiple Deprivation (produced by the Department for Communities and Local Government in 2011). This is a UK-wide indicator, by which areas are ranked according to a combination of employment, health, education, housing, and other factors.

We also use data concerning London's retail centres, as defined by the Department for Communities and Local Government (see www.planningstatistics.org.uk). These are consistently-defined 'areas of town centre activity', and measurements of the total area of retail floorspace are given for each; it is this quantity that is used as a proxy for the size of each centre in our model. To calculate the distances between residential areas and retail centres required by our model, the Euclidean distance between zone centroids is used.

\section{Numerical simulations. The data described above are used as the inputs for} numerical simulations of the model described previously. The model is implemented as a discrete-time system, with each time step involving sequential iteration through the model equations given in the earlier text.

In order to obtain the configuration used for the demonstration case, a parameter search was carried out across the space of all tunable parameters. The process began by selecting plausible ranges for each parameter, informed by previous work with similar models. One simulation was carried out for each configuration in a coarse sampling of this space, with several observables computed for each completed run: the distribution of riot magnitude across all sites, the number of riot sites where the level of offending was of an order higher than the mean level, the temporal progression of the simulation, and the distribution of flow-weighted travel distances. Using these observations, a smaller region of parameter space was identified for which all observations were of similar character to the riot data (in the sense that their relative difference was within a certain tolerance). The process was then repeated for the smaller parameter space, using a lower tolerance, and several further similar iterations followed.

1. Colizza, V., Barrat, A., Barthélemy, M. \& Vespignani, A. The role of the airline transportation network in the prediction and predictability of global epidemics. Proc. Natl. Acad. Sci. USA 103, 2015-2020 (2006).

2. Balcan, D. et al. Multiscale mobility networks and the spatial spreading of infectious diseases. Proc. Natl. Acad. Sci. USA 106, $21484-21489$ (2009).

3. Johansson, A. et al. Crowd and environmental management during mass gatherings. Lancet Infect. Dis. 12, 150-156 (2012).

4. Kinney, R., Crucitti, P., Albert, R. \& Latora, V. Modeling cascading failures in the North American power grid. Eur. Phys. J. B 46, 101-107 (2005).

5. Metropolitan Police. Operation Kirkin Strategic Review: Interim Report (2011). URL http://content.met.police.uk/News/August-Disorder--Police-InterimReport/1400005002176/1257246745756. Last accessed 12th December 2012.

6. Gross, M. Why do people riot? Curr. Biol. 21, 673-676 (2011)

7. Riots Communities and Victims Panel. 5 Days In August: An Interim Report On The 2011 English Riots (2011). URL http://riotspanel.independent.gov.uk/
wp-content/uploads/2012/04/Interim-report-5-Days-in-August.pdf. Last accessed 12th December 2012.

8. The Guardian and LSE. Reading The Riots: Investigating England's Summer Of Disorder (2011). URL http://www.guardian.co.uk/uk/series/reading-the-riots. Last accessed 12th December 2012.

9. Haddock, D. \& Polsby, D. Understanding riots. Cato J. 14, 147-157 (1994).

10. HMIC. The Rules Of Engagement: A Review Of The August 2011 Disorders (2011) URL http://www.hmic.gov.uk/media/a-review-of-the-august-2011-disorders20111220.pdf. Last accessed 12th December 2012.

11. Metropolitan Police. 4 Days In August: Strategic Review into the Disorder of August 2011 (2012). URL http://content.met.police.uk/News/MPS-report-into-summerdisorder/1400007360193/1257246745756. Last accessed 12th December 2012.

12. Yarwood, R. The geographies of policing. Prog. Hum. Geog. 31, 447-465 (2007). 13. Schelling, T. C. Dynamic models of segregation. J. Math. Sociol. 1, 143-186 (1971).

14. Cederman, L.-E. Emergent Actors in World Politics: How States and Nations Develop and Dissolve (Princeton University Press, Princeton, 1997).

15. Siegel, D. A. Social networks and collective action. Am. J. Polit. Sci. 53, 122-138 (2009).

16. Mason, T. D. Individual participation in collective racial violence: A rational choice synthesis. Am. Polit. Sci. Rev. 78, 1040-1056 (1984).

17. McPhail, C. The Myth Of The Madding Crowd (Aldine, New York, 1991).

18. Auyero, J. \& Moran, T. P. The dynamics of collective violence: Dissecting food riots in contemporary Argentina. Soc. Forces 85, 1341-1367 (2007).

19. Shaw, C. \& McKay, H. Juvenile Delinquency And Urban Areas: A Study OfRates Of Delinquency In Relation To Differential Characteristics Of Local Communities In American Cities (University of Chicago Press, Chicago, 1969).

20. Cornish, D. B. \& Clarke, R. V. The Reasoning Criminal: Rational Choice Perspectives on Offending (Springer-Verlag, New York, 1986).

21. Baudains, P., Braithwaite, A. \& Johnson, S. D. Target choice during extreme events: A discrete spatial choice model of the 2011 London riots. Criminology (forthcoming).

22. McPhail, C. The dark side of purpose: Individual and collective violence in riots. Sociol. Quart. 35, 1-32 (1994).

23. Brantingham, P. L. \& Brantingham, P. J. Nodes, paths and edges: Considerations on the complexity of crime and the physical environment. J. Environ. Psychol. 13, 3-28 (1993).

24. Townsley, M. \& Sidebottom, A. All offenders are equal, but some are more equal than others: Variation in journeys to crime between offenders. Criminology 48, 897-917 (2010)

25. Wortley, R. A classification of techniques for controlling situational precipitators of crime. Secur. J. 14, 63-82 (2001).

26. Epstein, J. M. Modeling civil violence: An agent-based computational approach. Proc. Natl. Acad. Sci. USA 99, 7243-7250 (2002).

27. Granovetter, M. Threshold models of collective behavior. Am. J. Sociol. 83, 14201443 (1978).

28. Myers, D. J. The diffusion of collective violence: Infectiousness, susceptibility, and mass media networks. Am. J. Sociol. 106, 173-208 (2000).

29. Sampson, R. J., Raudenbush, S. W. \& Earls, F. Neighborhoods and violent crime: A multilevel study of collective efficacy. Science 277, 918-924 (1997).

30. Kirkland, J. A. \& Maciejewski, A. A. A simulation of attempts to influence crowd dynamics. In Proc. IEEE Intl. Conf. on Syst., Man, and Cybern. 2003 5, 4328-4333 (2003).

31. Bhat, S. S. \& Maciejewski, A. A. An agent-based simulation of the L.A. 1992 riots. In Proc. 2006 Intl. Conf. Artif. Intell. 1, 76-79 (2006).

32. Yiu, S. Y., Gill, A. \& Shi, P. Investigating strategies for managing civil violence using the MANA agent-based distillation. In Proc. Land Warfare Conf. 2002 (2002).

33. Goh, C. K., Quek, H. Y., Tan, K. C. \& Abbass, H. A. Modeling civil violence: An evolutionary multi-agent, game theoretic approach. In Proc. IEEE Congr. Evol. Comput. 2006, 1624-1631 (2006).

34. Weidmann, A. G. \& Salehyan, I. Violence and Ethnic Segregation: A Computational Model Applied to Baghdad. Intl. Stud. Quart. (forthcoming). Available online at: http://nils.weidmann.ws/publications/weidmann13baghdad. Last accessed 12th December 2012.

35. Torrens, P. M. \& McDaniel, A. W. Modeling geographic behavior in riotous crowds. Ann. Assoc. Am. Geogr. 103 (2013).

36. Wilkinson, S. I. Riots. Ann. Rev. Polit. Sci. 12, 329-343 (2009).

37. Home Office. An Overview Of Recorded Crimes And Arrests Resulting From Disorder Events In August 2011 (2011). URL http://www.homeoffice.gov.uk/ publications/science-research-statistics/research-statistics/crime-research/ overview-disorder-aug2011/. Last accessed 12th December 2012.

38. Clauset, A., Shalizi, C. R. \& Newman, M. E. J. Power-law distributions in empirical data. SIAM Rev. 51, 661-703 (2009).

39. Wilson, A. G. Entropy In Urban And Regional Modelling (Pion, London, 1970). 40. Wilson, A. G. The Science OfCities And Regions: Lectures On Mathematical Model Design (Springer, Berlin, 2012).

41. Ben-Galim, D. \& Gottfried, G. Exploring The Relationship Between Riot Areas And Deprivation: An IPPR Analysis (2011). URL http://www.ippr.org/articles/56/ 7857. Last accessed 12th December 2012.

42. Wilson, A. G. A family of spatial interaction models, and associated developments. Env. Plan. 3, 1-32 (1971). 
43. Harris, B. \& Wilson, A. G. Equilibrium values and dynamics of attractiveness terms in production-constrained spatial-interaction models. Env. Plan. A 10, 371-388 (1978).

44. Wilson, A. G. Boltzmann, Lotka and Volterra and spatial structural evolution: an integrated methodology for some dynamical systems. J. R. Soc. Interface 5, 865871 (2008).

45. Anderson, R. M. \& May, R. M. Infectious Diseases Of Humans: Dynamics And Control (Oxford University Press, Oxford, 1992).

46. McFadden, D. L. Econometric analysis of qualitative response models. Handbook of Econometrics 2, 1395-1457 (1984).

\section{Acknowledgements}

We acknowledge the financial support of the Engineering and Physical Sciences Research Council (EPSRC) under the grant ENFOLD-ing - Explaining, Modelling, and Forecasting Global Dynamics, reference EP/H02185X/1, and the Security Science Doctoral Training Centre, reference EP/G037264/1. We are grateful for the assistance of the Metropolitan Police in the provision of offence data, and thank S. Johnson and P. Baudains for critical discussions. We also thank the anonymous reviewer for his/her particular helpful comments.

\section{Author contributions}

T.P.D. and H.M.F. analysed data, formulated the model, carried out numerical simulations and wrote the paper. A.G.W. formulated the model, oversaw the analysis and wrote the paper. S.R.B. contributed to model development and the writing of the paper.

\section{Additional information}

Competing financial interests: The authors declare no competing financial interests.

License: This work is licensed under a Creative Commons

Attribution-NonCommercial-ShareALike 3.0 Unported License. To view a copy of this license, visit http://creativecommons.org/licenses/by-nc-sa/3.0/

How to cite this article: Davies, T.P., Fry, H.M., Wilson, A.G. \& Bishop, S.R. A mathematical model of the London riots and their policing. Sci. Rep. 3, 1303; DOI:10.1038/ srep01303 (2013) 